\title{
Prediction of Arrival Profiles and Queue Lengths Along Signalized Arterials by Using a Markov Decision Process
}

\author{
Nikolaos Geroliminis and Alexander Skabardonis
}

\begin{abstract}
An analytical methodology for prediction of the platoon arrival profiles and queue length along signalized arterials is proposed. Traffic between successive traffic signals is modeled as a two-step Markov decision process (MDP). Traffic dynamics are modeled with the use of the kinematic wave theory. The MDP formulation allows prediction of the arrival profiles several signals downstream from a known starting flow. This modeling approach can be used to estimate queue lengths and predict travel times, even in cases in which data from loop detectors are unknown, inaccurate, or aggregated. The proposed model was applied to two real-world test sites. The queues estimated with the model are in close agreement with the results from microscopic simulation.
\end{abstract}

A significant proportion of travel takes place on urban arterials controlled by traffic signals. Monitoring of system performance on arterials and evaluation of alternative traffic management strategies require analysis tools for accurate estimation of the queue lengths at the intersection approaches, the travel times on the arterial links, and other measures. A number of techniques have been developed to estimate arterial performance measures from surveillance data. Most of the proposed models, however, are quite site specific and cannot be readily applied to other locations.

There is a need for models that can be used to estimate travel times on arterial streets on the basis of conventional loop detector data (flow, speed, and occupancy). Such models should be based on realistic modeling of traffic dynamics to predict accurately the interaction of vehicles as they travel along the arterials and the queues formed at the signalized intersections. At the same time, such models should be robust and should be able to provide performance measures even with faulty or limited detector data.

The objective of the study described here is to develop an analytical model that can be used to estimate platoon dispersion on arterial links and queues at traffic signals. This is an important part of on ongoing study to develop a system for the online estimation of arterial travel times from surveillance data.

The paper first briefly reviews existing platoon dispersion models. Next, the model formulation for estimation of the platoon dispersion and queue lengths at signalized intersections is described. The last section presents the application of the proposed model on two test sites and outlines ongoing and future model enhancements.

Institute of Transportation Studies, University of California, Berkeley, 109 McLaughlin Hall, Berkeley, CA 94720-1720.

Transportation Research Record: Journal of the Transportation Research Board, No. 1934, Transportation Research Board of the National Academies, Washington, D.C., 2005, pp. 116-124.

\section{BACKGROUND}

The traffic departing a traffic signal initially moves as a tight platoon with short vehicle headways. This platoon tends to disperse the farther downstream that it travels because of differences in vehicle speeds, vehicle interactions (lane changing and merging), and other interferences (parking, pedestrians, and other frictional effects) (1). Prediction of platoon size and dispersion is important for determination of the traffic arrivals at downstream intersections to assess the need for signal coordination and to optimize the signal timing plans in coordinated signal systems.

Pacey modeled platoon dispersion by assuming that the speed of any single vehicle traveling on an arterial link is constant and unrestricted overtaking (2). He derived the travel time distribution, $g(\tau)$, along the arterial segment by also assuming that the vehicle speeds are normally distributed:

$g(\tau)=\frac{D}{\tau^{2} \sigma \sqrt{2 \pi}} \exp \left[-\frac{\left(\frac{D}{\tau}-\bar{u}\right)^{2}}{2 \sigma^{2}}\right]$

where

$D=$ distance from the upstream signal to a downstream location where the vehicle arrivals are observed;

$\tau=$ individual vehicle travel time along distance $D$; and

$\bar{u}$ and $\sigma=$ mean and standard deviation of vehicle speeds, respectively.

The number of vehicles passing the downstream observation point (Location 2) in the time interval $(t, t+d t)\left[q_{2}\left(t_{2}\right) d t_{2}\right]$ is

$q_{2}\left(t_{2}\right) d t_{2}=\int_{l_{1}} q_{1}\left(t_{1}\right) g\left(t_{2}-t_{1}\right) d t_{1} d t_{2}$

where $q_{1}\left(t_{1}\right) d t_{1}$ is the number of vehicles passing the upstream signal (Location 1$)$ in the interval $(t, t+d t)$, and $g\left(t_{2}-t_{1}\right)$ is the probability density function of travel time $\left(t_{2}-t_{1}\right)$ according to Equation 1.

Robertson's formula (3), implemented in the TRANSYT simulation and optimization model, is the most well-known model of platoon dispersion. It is similar to the discrete version of Pacey's model, but it has been derived by assuming a geometric distribution of vehicle travel times:

$q_{2}(i+t)=\frac{1}{1+\alpha t} q_{1}(i)+\left(1-\frac{1}{1+\alpha t}\right) q_{2}(i+t-1)$ 
where

$$
\begin{aligned}
t & =\text { average travel time and is equal to } 0.8, \\
\alpha & =\text { platoon dispersion factor, and } \\
i & =\text { time step. }
\end{aligned}
$$

Robertson's model has shown satisfactory agreement with field data under undersaturated flow conditions. Its main advantage over Pacey's model is the much lower computational requirements, which is a critical issue in signal timing optimization for large networks (4). In the TRANSYT model, a flow histogram of traffic leaving the stop line of the upstream signal is first constructed (departure profile), and it is transformed by using Equation 3 to obtain the arrival profile at the downstream signal. The rate of platoon dispersion depends on the platoon dispersion factor, $\alpha$; the default value is 0.35 in the TRANSYT model. Typical values of $\alpha$ range from 0.25 (tight platoons typical of suburban high-speed arterials) to 0.5 (dispersed platoons typical of downtown areas). However, the arrival profiles are constructed under the assumption that traffic arrivals are not obstructed by the queue presence at the traffic signal. Thus, the model cannot accurately estimate the spatial extent of the queues. Also, Rumsey and Hartley reported that Robertson's model predicts a greater dispersion of the platoon for any given mean travel time because of the longer tail of the geometric distribution than the corresponding transformed normal distribution (5).

\section{MODEL FORMULATION}

Traffic behavior between successive traffic signals is modeled by a one-step recursive formulation with some special characteristics. This one-step recursive formulation is defined as follows: consider a system in state $i$ at time $t$ with the property that, given the present state, the future is conditionally independent of the past. The state of the system at time $t+1$ is then predicted from the state of the system at time $t$. This approach is very similar to the approach that uses the memoryless property of a Markov decision process (MDP).

Initially, the arrivals of vehicles at the downstream traffic signal $(i+1)$ are a function $f$ of the departures of vehicles from upstream intersection $i$. Sequentially, the departures of the vehicles from the downstream signal are a function $h$ of the arrivals at this intersection. The procedure's diagram for predicting the platoon size and shape between two adjacent signals is as follows:

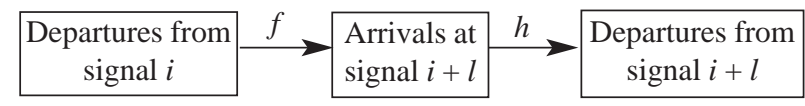

Mathematically, this process is expressed by the formulas

$$
\begin{aligned}
& q_{i+1, j}^{\text {in }}\left(t^{\prime}\right)=f\left[q_{i, j}^{\text {out }}(t)\right] \\
& q_{i+1, j}^{\text {out }}\left(t^{\prime}\right)=h\left[q_{i+1, j}^{\text {in }}\left(t^{\prime}\right)\right]
\end{aligned}
$$

where

$$
\begin{aligned}
q_{i+1, j}^{\mathrm{in}}(t)= & \text { arriving flow at signal } i+1 \text { at time } t \text { departing from } \\
& \text { signal } i \text { during cycle } j, \\
q_{i, j}^{\text {out }}(t)= & \text { departing flow from signal } i \text { at time } t \text { during cycle } j, \\
t^{\prime}= & \text { arrival time of a vehicle at the downstream signal } \\
& \text { traveling at free-flow speed, and } \\
h= & \text { signal filter function }
\end{aligned}
$$

$t^{\prime}=t+\frac{L}{u_{f}}$

where

$$
\begin{aligned}
L & =\text { signal spacing }, \\
u_{f} & =\text { free-flow speed }, \text { and } \\
f & =\text { platoon dispersion function }
\end{aligned}
$$

Thus, by alternating the platoon dispersion process with the signal filter one, the arrival profiles at several intersections downstream can be predicted from a known starting flow. A schematic representation of the algorithm is shown in Figure 1. This formulation can be used to predict travel times and estimate queue lengths, even in cases in which traffic flow data from loop detectors are unknown, inaccurate, or aggregated.

In the case of incomplete information regarding the signal settings (e.g., actuated signals with variable green times), the formulation has stochastic characteristics and is an MDP. This process has the Markov property, as given the present arrivals $A_{n}$ from signal $n$, the distribution of $A_{n+1}$ is determined. This can be restated as the probability that $A_{n+1}$, which is conditional on the whole past history of the sequence $\left(A_{m}=i_{m}\right)_{m=1}^{n}$ (where $m$ is an index and $m=1$ to $n$ indicates the arrivals for all signals upstream of $n+1$ ), reduces to a probability of $A_{n+1}$ conditional on the latest value alone, $A_{n}$. Given that the signal settings are fixed, MDP is equivalent to a one-step recursion, as shown above.

$\mathbf{P}\left[A_{n+1}=i_{n+1} \mid\left(A_{m}=i_{m}\right)_{m=1}^{n}\right]=\mathbf{P}\left(A_{n+1}=i_{n+1} \mid A_{n}=i_{n}\right)$

where $\mathbf{P}$ is probability, and $i_{n}$ is the state (arrival's profile) at signal $n$.

\section{Estimation of Platoon Dispersion}

Platoon dispersion was modeled by using the kinematic wave theory proposed by Lighthill and Whitham (6) and Richards (7) (the LWR theory). The main postulate of the LWR theory is that a functional relationship exists between the traffic flow $(q)$ and the traffic density $(k)$ and that this relationship could be used to describe the speed at which a change in traffic flow propagates either downstream or upstream from an origin point. Shock waves are generated by the traffic signal, which causes congested conditions to develop near the stop line during the red time and capacity conditions to occur when the queue is discharging at the saturation flow rate. The first vehicle departs from the stop line at the free-flow speed, but because of interfaces between different points of the $q-k$ diagram, the following vehicles depart from the stop line at slower speeds. The level of dispersion in speeds and, as a result, in the platoon depends on the curvature of the increasing part of the $q-k$ diagram.

The proposed methodology estimates the average platoon dispersion ratio and the nonuniform platoon profile for any concave $q-k$ relationship. In this paper, it is assumed that the $q-k$ diagram has the quadratic form, as shown in Figure 2, so numerical results can be obtained. The form of this curve is based on data in the 2000 Highway Capacity Manual (8) for undersaturated traffic conditions.

\section{Single Platoon}

The analysis is based on the conservation law of flow. The flow decreases with the distance from the intersection stop line, but the 


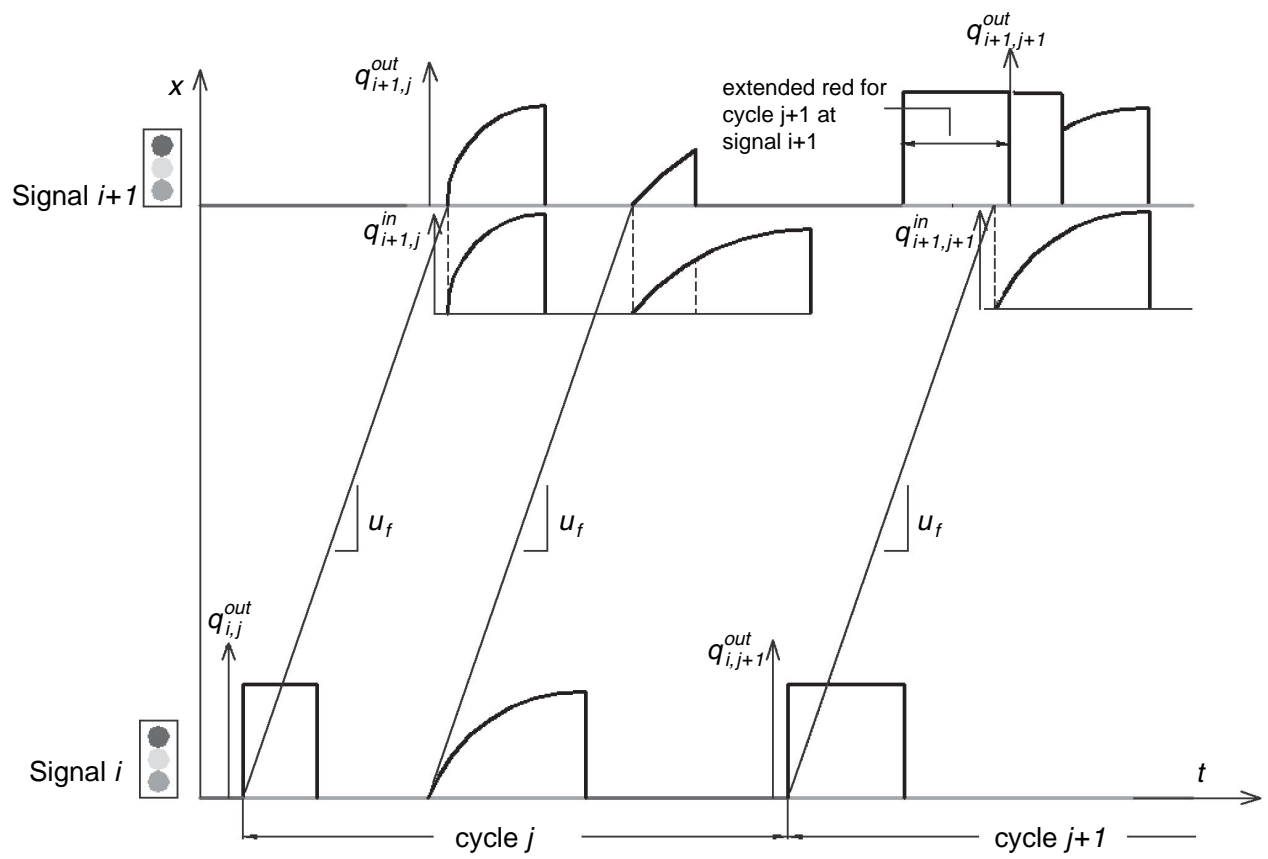

FIGURE 1 Schematic representation of recursion process.

number of vehicles does not change. When the flow is moving from Traffic State A (decreasing part of $q-k$ diagram) to Traffic State E (increasing part of diagram), this cannot be accomplished with a linear interface AE but should follow intermediate States B, C, and D and the route $\mathrm{A} \rightarrow \mathrm{B} \rightarrow \mathrm{C} \rightarrow \mathrm{D} \rightarrow \mathrm{E}$ (Figure $3 a$ ). The time-space diagram in Figure $3 b$ illustrates the behavior of vehicles departing from the stop line, where

$L_{i}=$ distance between the stop line and the section of the trajectory of the last vehicle with the $i$ th interface,

$t_{i}^{\prime}=$ maximum horizontal distance between the $(i+1)$ th and the $i$ th interfaces,

$t_{i}=$ platoon width at distance $L_{i}$ from the intersection stop line (s), and

$q_{i}=$ flow between the $(i+1)$ th and the $i$ th interfaces.

The number of vehicles $\left(N_{\text {tot }}\right)$ departing from the intersection stop line is

$$
N_{\text {tot }}=q_{0} \cdot t_{0}
$$

where $q_{0}$ is the initial platoon flow and $t_{0}$ is the width at the intersection stop line. From the geometry of Figure 3, it is easy to compute $L_{i}$ and $t_{i}$

$t_{i}=t_{0}+\frac{L_{i}}{u_{0}}-\frac{L_{i}}{u_{f}}$

$L_{i}=t_{0} \cdot \frac{\left.u_{0} \cdot \frac{\partial q}{\partial k}\right|_{\mathrm{BC}}}{u_{0}-\left.\frac{\partial q}{\partial k}\right|_{\mathrm{BC}}}$

where

$u_{f}=$ free-flow speed;

$u_{i}=$ speed of the group of vehicles between the $i$ th and the $(i+1)$ th interface; for $i$ equal to 0 this is the speed at capacity (where flow is equal to capacity); and

$\left.\frac{\partial q}{\partial k}\right|_{\mathrm{BC}}=$ speed of the interface $\mathrm{B} \rightarrow \mathrm{C}$ in Figure $3 a$.

$q$

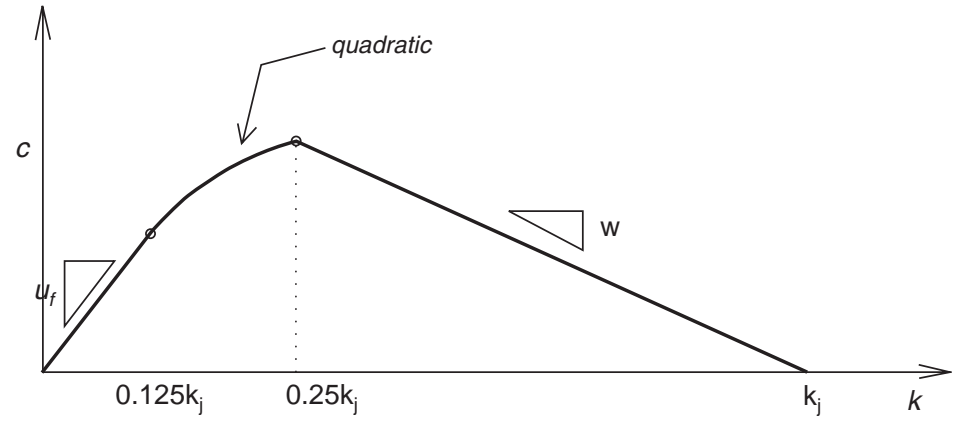

FIGURE 2 Assumed form of $q-k$ diagram ( $c=$ maximum flow; $w=$ congested wave speed]. 


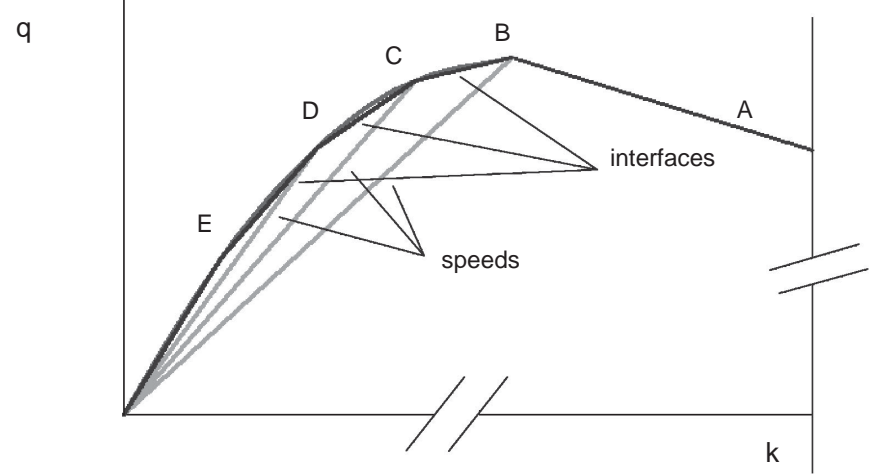

(a)

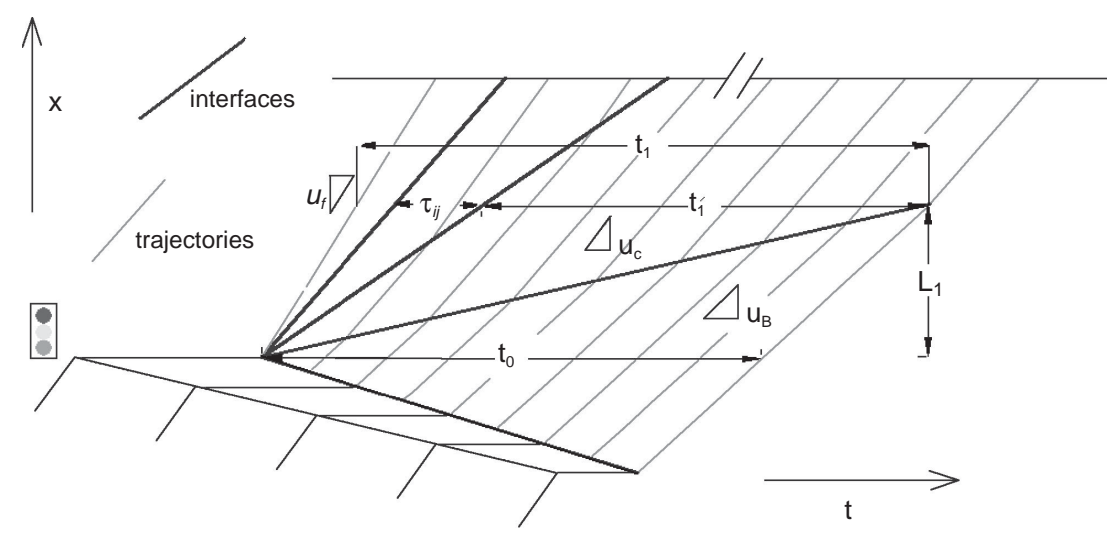

(b)

FIGURE 3 Estimation of platoon dispersion: (a) speed and interfaces in the $q-k$ diagram and $(b) x-t$ diagram for estimation of platoon dispersion.

For simplicity, the speed of the $i$ th interface is indicated as $q_{i}^{\prime}$. The platoon dispersion ratio $\left(r_{i}\right)$ at distance $L_{i}$ downstream from the signal is equal to

$r_{i}=\frac{q_{i}}{q_{0}}=\frac{\left(\frac{N_{\mathrm{tot}}}{t_{i}}\right)}{\left(\frac{N_{\mathrm{tot}}}{t_{0}}\right)}=\frac{t_{0}}{t_{i}}$

A numerical approach with finite differences was developed to estimate $t_{i}$ and $r_{i}$ at a distance $L_{i}$ from the intersection stop line (Figure 4). The finite differences equations are

$t_{i}=t_{i-1}+\frac{L_{i}-L_{i-1}}{u_{i-1}}-\frac{L_{i}-L_{i-1}}{u_{f}}$

$L_{i}-L_{i-1}=t_{i-1}^{\prime} \cdot \frac{q_{i-1}^{\prime} \cdot u_{i-1}}{u_{i-1}-q_{i-1}^{\prime}}$

$t_{i}^{\prime}=L_{i} \cdot \frac{q_{i}^{\prime}-q_{i-1}^{\prime}}{q_{i}^{\prime} \cdot q_{i-1}^{\prime}}$

The approach described above allows the average flow rate to be estimated as a function of distance $L$ from the beginning of the platoon. It should be mentioned that distance $L$ is not the distance from the first upstream signal. It shows the average arrival flow of vehicles at distance $L_{i}$ downstream of a signal where vehicles stopped because of the red phase. This methodology is expressed as a first-order approximation because the pattern of the dispersion is not estimated; just the average value of the arrival flow is estimated. To estimate the shape of the platoon arrivals (flow in a time-space region), the following should be added to Equations 11 to 14:

$$
\begin{aligned}
& \tau_{i j}\left(q_{j}, L_{i}\right)=\frac{L_{i}}{q_{j-1}^{\prime}}-\frac{L_{i}}{q_{j}^{\prime}} \quad \forall j: j \geq i \\
& \tau_{i n}\left(q_{n}, L_{i}\right)=\frac{L_{i}}{q_{n-1}^{\prime}}-\frac{L_{i}}{u_{f}}
\end{aligned}
$$

where $n$ is the number of steps in the finite differences procedure.

The time $\tau_{i j}$ in Equation 15 shows the amount of time in the platoon pattern at distance $L_{i}$ downstream of the signal that the flow is equal to $q_{j}$. Therefore, Figure $3 b$ shows that

$\sum_{j=i}^{j=n} \tau_{i j}\left(q_{j}, L_{i}\right)=t_{i}$

and

$\tau_{i j}=t_{i}^{\prime}$

Figure 5 shows the results of the finite differences approach for a starting platoon of $20 \mathrm{~s}$ in width departing at capacity [1,800 vehicles per hour (veh/h)] from the stop line and under the assumption of a quadratic form for the increasing part of the $q-k$ diagram with a free-flow 


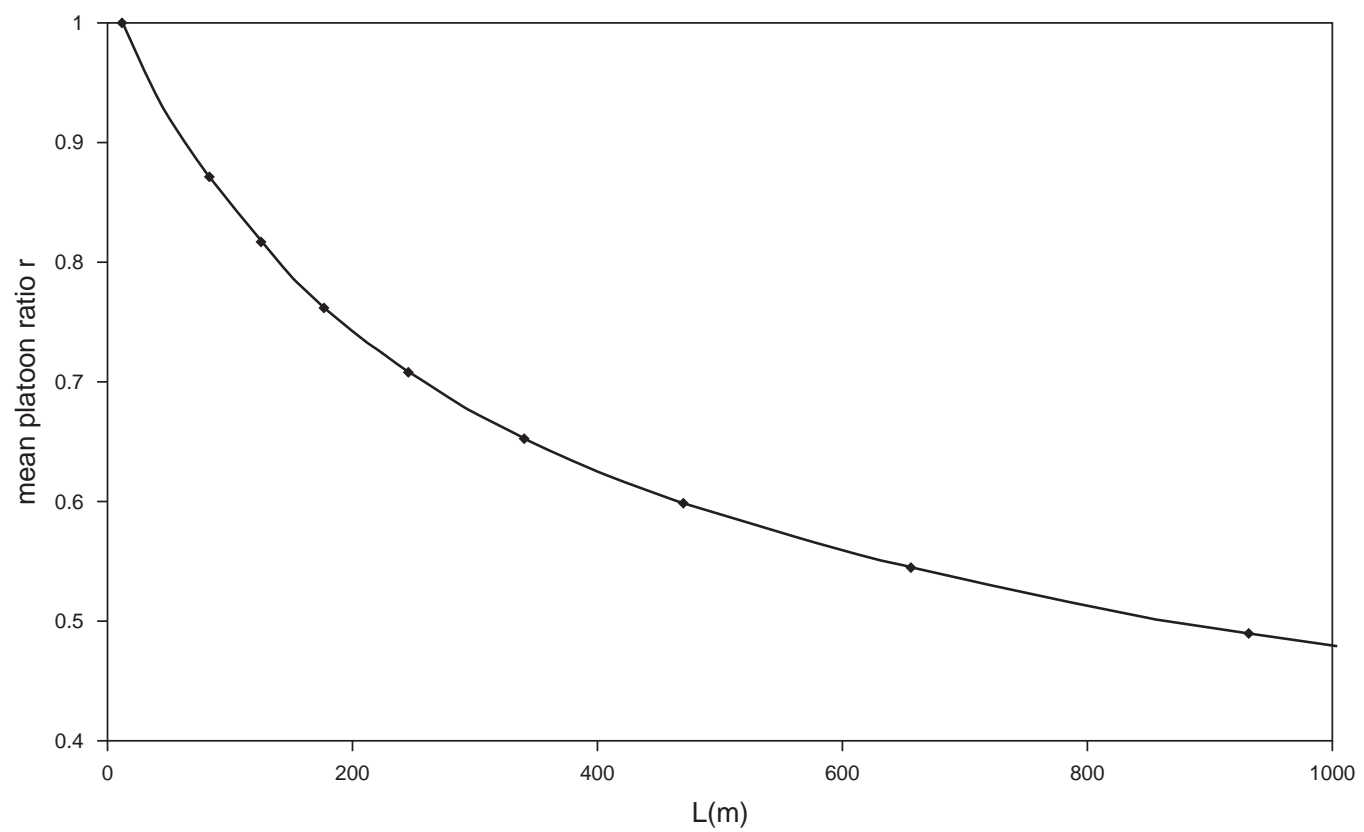

FIGURE 4 Mean platoon ratio versus distance $L$ from intersection stop line.

speed $\left(u_{f}\right)$ of $30 \mathrm{mph}$ and a saturation speed (speed at capacity; $u_{s}$ ) of $18 \mathrm{mph}$.

\section{Interaction of Platoons}

When two distinct vehicle platoons, e.g., the arterial through platoon and the turning platoon from the side streets on the arterial, depart from the upstream intersection at different times (because of different signal phases), the second platoon gets closer to the first platoon as traffic travels downstream. This is because the last vehicles of the first platoon travel at slower speeds than the first vehicles of the second platoon. As the distance $L$ from the upstream intersection increases, more vehicles from the second platoon join the first platoon and a single merged platoon forms. The rate at which the second platoon joins the first one $(d t / d L)$ expresses how much time (in

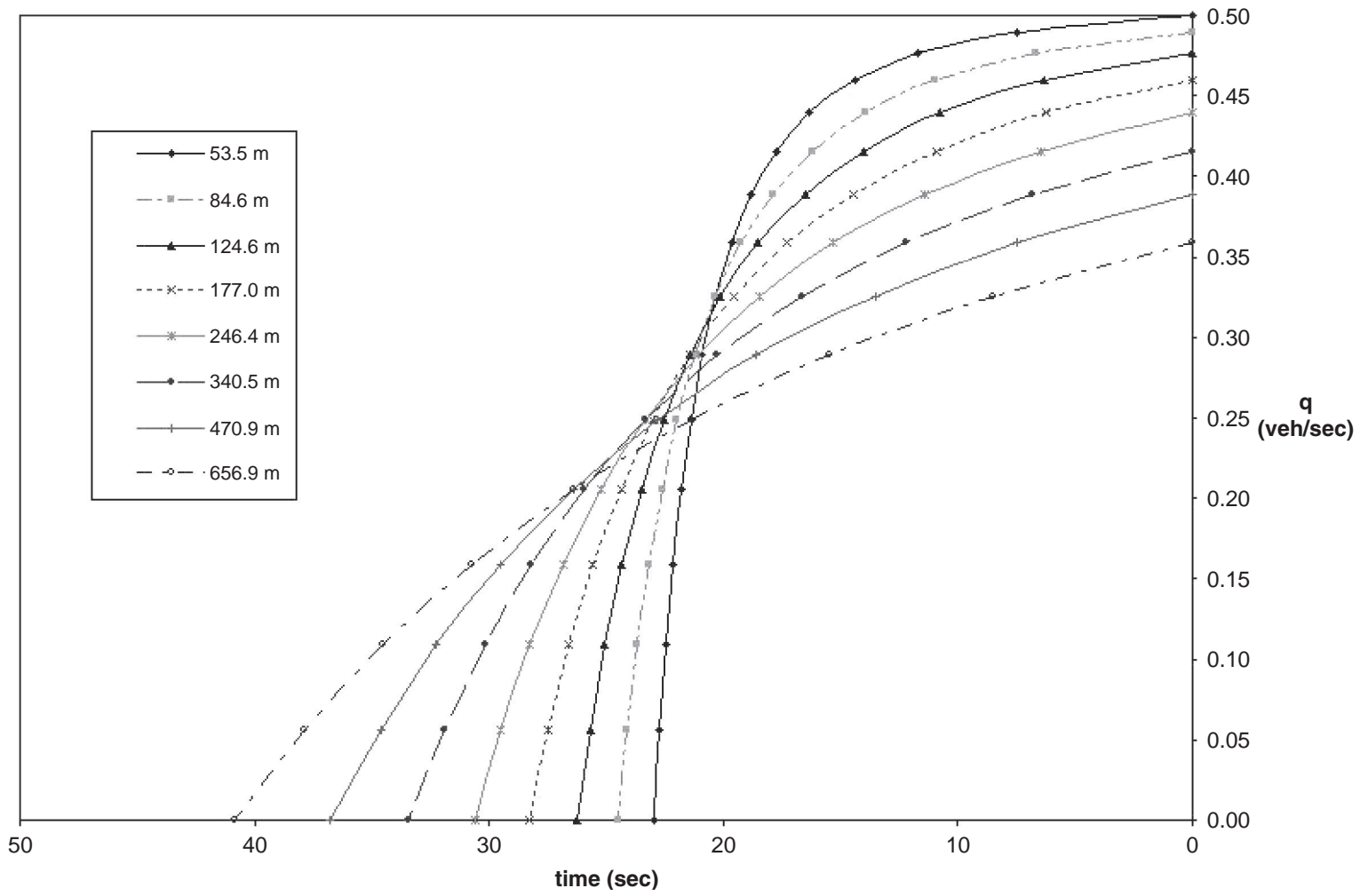

FIGURE 5 Platoon pattern as function of distance $L$. 
seconds) that the first platoon spreads out as $L$ increases and is given by the formula

$\left.\frac{d t}{d L}\right|_{L=L_{i}}=\frac{1}{v_{0,2}}-\frac{1}{v}=\frac{k_{0,2}}{q_{0,2}}-\frac{k_{n, 1}-k_{0,2}}{q_{n, 1}-q_{0,2}}=\frac{\left(\frac{q_{n, 1}}{v_{0,2}}-k_{n, 1}\right)}{q_{n, 1}-q_{0,2}}$

where

$$
\begin{aligned}
v_{0,2}, k_{0,2}, q_{0,2}= & \text { speed, density, and flow of the first group of the } \\
& \text { second platoon, respectively; } \\
k_{n, 1}, q_{n, 1}= & \text { density and flow of the last group of the first platoon, } \\
& \text { respectively; and } \\
\frac{q_{n, 1}-q_{0,2}}{k_{n, 1}-k_{0,2}}= & \text { interface between the flow of the last group of } \\
& \text { the first platoon and the first group of the second } \\
& \text { platoon. }
\end{aligned}
$$

This formula has been derived by considering the fact that headway $(d t)$ and spacing $(d L)$ are the reciprocal of flow and density, respectively. The numerator of this fraction expresses the time between two consequent arrivals of vehicles of the second platoon; i.e., it is the reciprocal of the rate at which fast vehicles pass a slow vehicle (9).

\section{Heterogeneous Drivers}

The model formulation described above by using the LWR theory for a nonlinear (concave) $q-k$ relationship predicts the dispersion in front of an isolated platoon, as well as the interactions and the dispersion of multiple platoons. However, as shown in Figure 5, the dispersion in the back (tail) of the platoon cannot be estimated.

The proposed platoon dispersion model is extended to estimate the dispersion of the back of the platoon by considering the heterogeneity in the drivers' behaviors. It is assumed that a portion of the drivers in the tail of the platoon follow larger discharge headways (i.e., their behavior is modeled with a $q-k$ diagram with a lower capacity). The back of the platoon can also be estimated in undersaturated conditions in which the leading vehicles of the platoon discharge at the saturation flow and the rest of the vehicles arrive and depart at the flow rate during the green time.

Figure 6 shows the results of the finite differences approach developed in the previous section by taking into consideration the heterogeneity of the drivers. The starting platoon of $35 \mathrm{~s}$ leaves the stop line; the leading platoon of $20 \mathrm{~s}$ in width follows the $q-k$ diagram in Figure 1 (capacity $=1,800 \mathrm{veh} / \mathrm{h}, u_{f}=30 \mathrm{mph}$, and $u_{s}=20 \mathrm{mph}$ ), the following platoon of $10 \mathrm{~s}$ departs at a rate of $1,500 \mathrm{veh} / \mathrm{h}$ (the same $q-k$ diagram), and the end of the platoon consists of slow vehicles that follow a different $q-k$ diagram (capacity at 1,200 veh/h and $u_{s}=15$ $\mathrm{mph}$ ). Figure 6 shows that the tail of the platoon is more dispersed compared with the dispersal results from the application of the original model shown in Figure 5.

\section{Prediction of Queue Lengths at Traffic Signal}

By using the recursive formulation, the LWR theory was applied to estimate the queue length and the flow profile departing from the intersection stop line. The effect of the signal is called the "signal filter." The process is illustrated in Figure 7.

Figure $7 a$ shows the vehicle platoon arriving at the intersection stop line in the form of a step function. Each step of the platoon is a group of vehicles whose traffic state (flow $q_{i}$ and speed $u_{i}$ ) and time width $\Delta t$ are estimated from the platoon dispersion model described above.

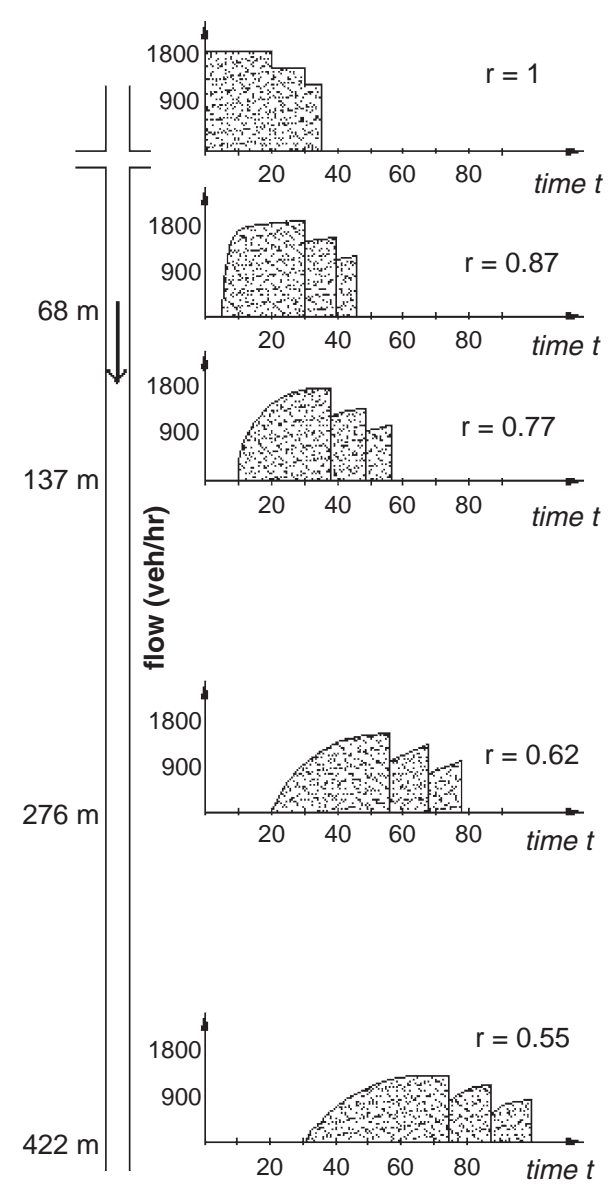

FIGURE 6 Platoon pattern versus distance $L$ considering heterogeneity in driver behavior.

If a group of vehicles approaches the traffic signal during the green time and no queue is present, it continues to move without any interaction. This movement follows the platoon dispersion approach described above and is not affected by the presence of the signal.

If the signal is red as the vehicles approach the intersection stop line, then these vehicles stop and the resulting shock wave between the existing traffic state and the state of the jam density is estimated by using the assumed $q-k$ curve. The process repeats for the next group of vehicles that stops at the signal (Figures $7 c$ and $7 d$ ). When the signal turns green, the queued vehicles discharge at the saturation flow. The recovery shock wave has speed $w$, the congested wave speed, which is the line in the $q-k$ diagram connecting the points of jam density and capacity flow (Figure $7 d$ ). When the queue has dissipated, the rest of the platoon that arrives during the green time clears the intersection without any interference from the traffic signal.

The proposed method predicts the time that the traffic signal starts serving the groups of uninterrupted vehicles; i.e., it predicts the effective extension of the red time because of the discharge of the queued vehicles. This is the time interval $\left(t_{5}\right.$ to $\left.t_{3}\right)$ in Figure $7 b$.

Each group of the platoon that stops because of the red phase effectively extends the red time $\left(t_{\text {ext.red }}\right)$ by the following amount:

$$
\begin{aligned}
t_{\text {exi,red }}^{i}\left(t, t+\Delta t_{i}\right)= & \min \left[\frac{N_{r}^{i}\left(t, t+\Delta t_{i}\right) \cdot L_{s}}{w}\right. \\
& \left.+\frac{N_{r}^{i}\left(t, t+\Delta t_{i}\right) \cdot L_{s}}{u_{s}}, g-t\right]
\end{aligned}
$$




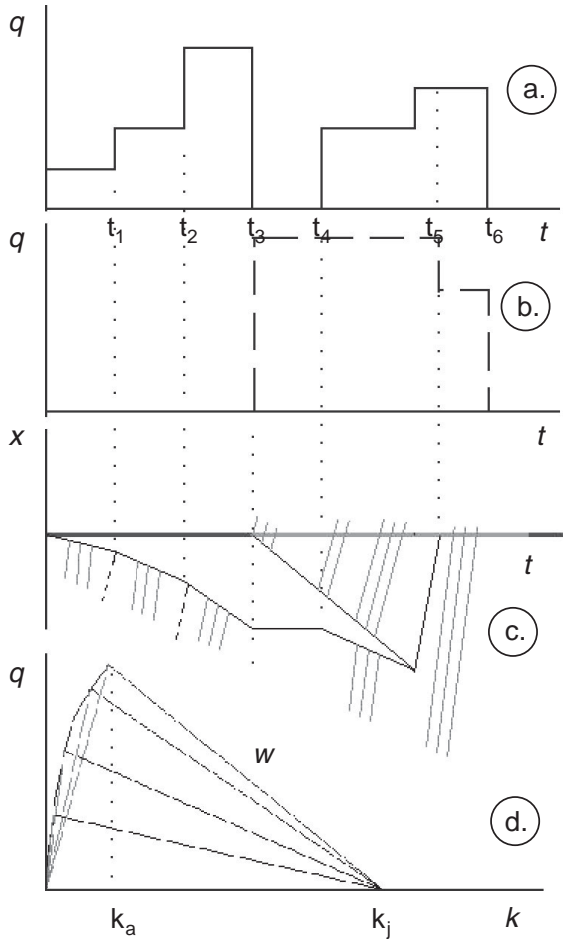

FIGURE 7 Platoon arrivals and departures at a traffic signal: (a) platoon arriving at intersection stop line; $(b)$ platoon departing from intersection stop line; $(c)$ time-space diagram, queue formation, and queue dissipation; and $(d)$ interfaces of traffic states in the $q-k$ diagram.

where

$w=$ congested wave speed;

$u_{s}=$ saturation speed, which is approximately the interface between the free-flow speed state and the saturation state, at which the queue discharges;

$g=$ green time;

$t=$ time that the first vehicle of the platoon group would reach the stop line if there were no vehicles ahead; it is measured from the start of the green time, and it can be expressed as modulo $\left(t, g_{i}\right)$.

$L_{s}=$ effective length of a stopped vehicle; this is the reciprocal of jam density $k_{j}$;

$\Delta t_{i}=$ time width of platoon group $i$, as estimated from the platoon dispersion model; and

$N_{r}^{i}=$ number of vehicles from platoon group $i$ that stop because of the signal.

The first term in the parentheses of Equation 19 is the sum of two time intervals: (a) the extension of the red time because of the shock wave and $(b)$ the time that it takes a vehicle discharging from the queue to reach the intersection stop line. The second term in parentheses expresses the fact that the estimated extended red time cannot be larger than the remaining green time. A stopped group of vehicles will pass the stop line at a time equal to the sum for all the previous groups of the same cycle. An uninterrupted group that reaches a red or extended red phase stops, while a group that reaches the green phase travels without interference. This approach is equivalent to that in the continuous LWR theory.
Platoon groups for which the total extended red time is equal to the green time cannot be served during the cycle during which they arrived. In this case, a residual queue remains at the end of the green. The red time of the next cycle is extended by

$t_{\text {exi, red }}=\min \left[\frac{n(q) \cdot L_{s}}{w}+\frac{n(q) \cdot L_{s}}{u_{s}}, g\right]$

where $n(q)$ is the number of queued vehicles at the end of the previous green cycle. If $n(q)$ becomes sufficiently large, then the extended red time is equal to the green time and the number of queued vehicles is the sum of the arriving vehicles in the next cycle plus the unserved vehicles of the previous cycle. The unserved vehicles of the previous cycle are the queued vehicles of the previous cycle minus the number of vehicles for which the first term in parentheses in Equation 20 equals $g$. The queue length of the queue in the beginning of the green at cycle $k, n_{k}^{\prime}(q)$ is

$n_{k}^{\prime}(q)=n_{k}(q)-N_{1 k}+N_{2 k}$

where $N_{1 k}$ and $N_{2 k}$ are the number of arrived vehicles and the number of served vehicles during the green time in cycle $k$, respectively.

\section{Oversaturation}

When the queue length exceeds the available link length, then it blocks the outflow from the upstream signal and sometimes reduces its capacity to zero. In this case, the number of served vehicles can be approximated by the number of vehicles served at the downstream signal. Therefore, this oversaturated system is equivalent (regarding the service rate and queue lengths) to a system with the following characteristics: $(a)$ infinite capacity of the downstream link (i.e., no blocking of traffic) and $(b)$ green time equal to

$g_{\text {eff }_{i}}^{i+1}=\frac{N_{i}^{t+1}}{N_{\text {sat }}^{i}} \cdot g_{i}=\frac{N_{i}^{t+1}}{s_{i} \cdot g_{i}} \cdot g_{i}=\frac{N_{i}^{t+1}}{s_{i}}$

where

$g_{i}=$ measured green time of signal at link $i$,

$N_{\text {sat }}^{i}=$ maximum number of vehicles served by signal $i$ at one cycle,

$N_{i}^{t+1}=$ real number of vehicles served by signal $i$ at cycle $t+1$,

$g_{\text {eff } i}^{t+1}=$ real effective green time at signal $i$ at cycle $t+1$, and

$s_{i}=$ saturation flow at signal $i$ (vehicles per hour of green time).

\section{MODEL APPLICATION}

The proposed model was applied to two real-life arterials to estimate the queue length on each intersection approach. (a) M Street is a closely spaced arterial with eight signalized intersections located in Washington, D.C. The average signal spacing is $450 \mathrm{ft}$. All signals operate as two-phase fixed-time signals with a common cycle length of $60 \mathrm{~s}$. (b) The Lincoln Avenue study section is part of a major arterial in Los Angeles. There are seven signalized intersections. Signal spacing ranges from 450 to $1,200 \mathrm{ft}$. There are three through lanes with exclusive left-turn lanes. All the signals are multiphase actuated with a common cycle length of $120 \mathrm{~s}$. During the morning peak periods, the queues at Intersection 7 fill Link 6-7 and block the outflow from upstream Intersection 6.

The basic data on intersection geometrics, signal settings, and free-flow speeds were assembled from related studies. Traffic data on flow, speed, and occupancy were obtained from system loop detectors positioned approximately $250 \mathrm{ft}$ from the intersection stop 
line. The data were coded into the CORSIM microscopic simulation model (10), and the performance was simulated for a total of 80 signal cycles. The simulation output was first compared with field data (delays and travel times) to verify that the model reasonably replicates field conditions at the test sites. Next, the simulated queue lengths predicted by the proposed model and the simulation were compared.
The results for three intersection approaches shown in Figure 8 indicate that the proposed model produces accurate estimates of queue lengths. In most of the cases, the difference between the modelpredicted and the simulated queue lengths is less than four vehicles. The model also captures the observed blocking of traffic at upstream Signal 6 on the Lincoln Avenue network because of oversaturation at Intersection (Node) 7 (Figure 8b).

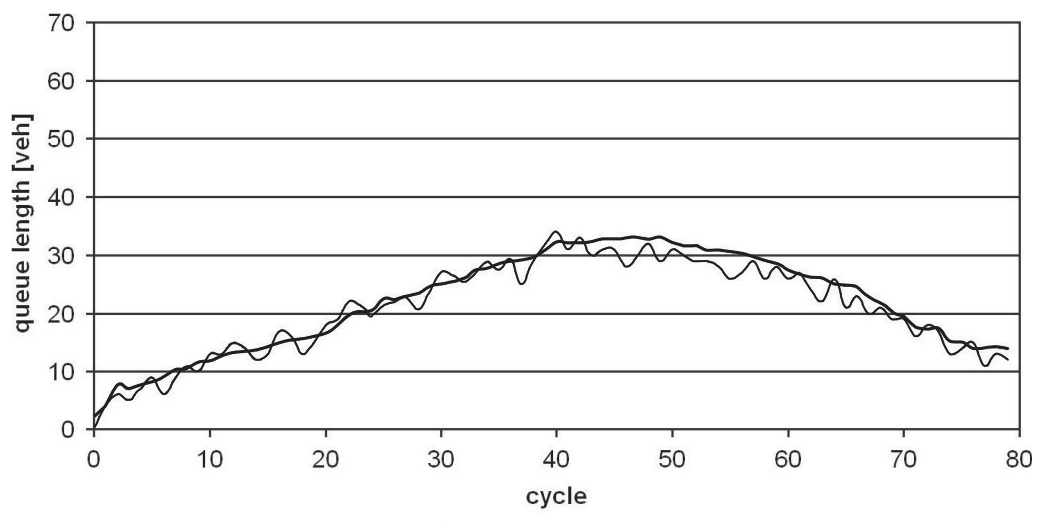

- Lq real - Lq est

(a)

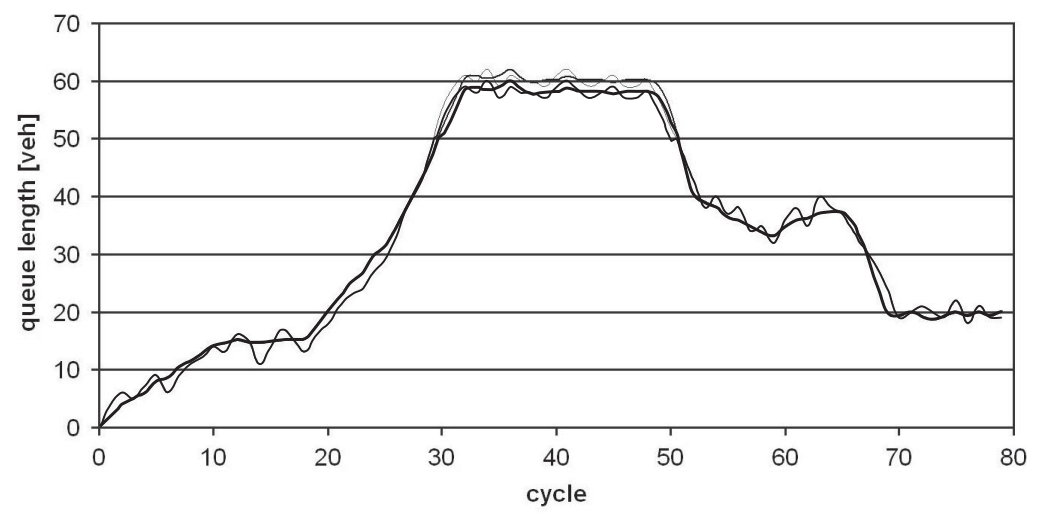

- Lq real - Lq est

(b)

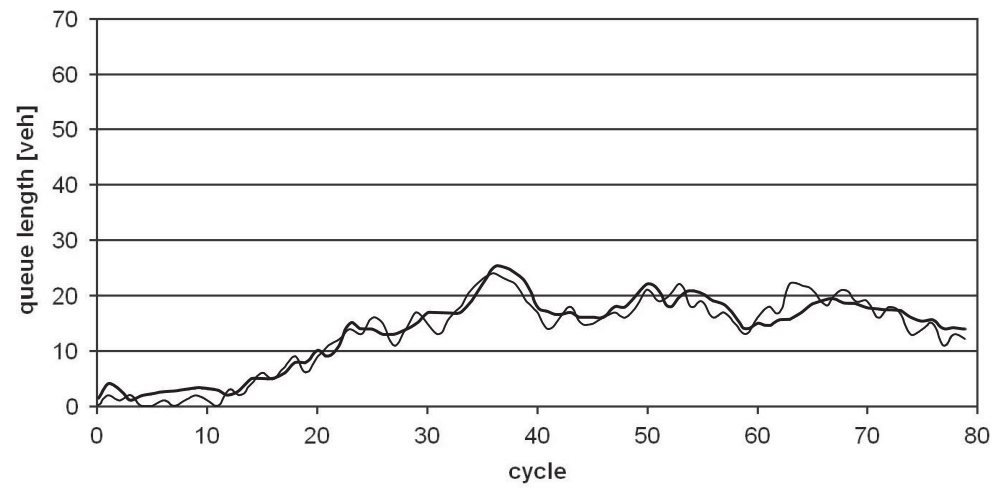

- Lq real - Lq est

(c)

FIGURE 8 Measured and estimated queue lengths: (a) Lincoln Street, Node 3;

(b) Lincoln Street, Node 7; (c) M Street, Node 2. 
The proposed model formulation based on the LWR theory realistically models the dispersion for both single and multiple platoons along arterials and explicitly considers the effect of residual queues at oversaturated intersections on the estimation of traffic arrivals.

The proposed recursive formulation permits the prediction of the arrival profiles many signals downstream from a known starting flow. This output is an important tool for the estimation of queue lengths and the prediction of link travel times even when the data from loop detectors are unknown, inaccurate, or aggregated. Work is in progress to extend the model to optimize the offsets between intersections in signalized networks and to estimate the travel times in real time along signalized arterials (11).

\section{ACKNOWLEDGMENTS}

The study described in this paper was performed as part of the California's Partners for Advanced Highways and Transit Program at the Institute of Transportation Studies, University of California, Berkeley. The authors thank Pravin Varaiya for comments and suggestions throughout the study.

\section{REFERENCES}

1. Denny, R. W., Jr. Traffic Platoon Dispersion Modeling. Journal of Transportation Engineering, Vol. 155, No. 2, 1989, pp. 193-207.
2. Pacey, G. M. The Progress of a Bunch of Vehicles Released from a Traffic Signal. Research Note Rn/2665/GMP. Road Research Laboratory, London, 1956.

3. Robertson, D. I. TRANSYT: A Traffic Network Study Tool. Road Research Laboratory Report LR 253. Road Research Laboratory, Crowthorne, United Kingdom, 1969

4. Rouphail, N., A. Tarko, and J. Li. Traffic Flow at Signalized Intersections. In Revised Monograph on Traffic Flow Theory, Chapter 9, 1992. http://www.tfhrc.gov/its/tft/ttt.htm. Accessed 2003.

5. Rumsey, A. F., and M. G. Hartley. Simulation of a Pair of Intersections Traffic Engineering \& Control, Vol. 13, 1972, pp. 522-525.

6. Lighthill, M. J., and J. B. Whitham. On Kinematic Waves. I. Flow Movement in Long Rivers. II. A Theory of Traffic Flow on Long Crowded Road. Proceedings of the Royal Society, A, Vol. 229, 1955, pp. 281-345.

7. Richards, P. I. Shockwaves on the Highway. Operations Research, Part B, Vol. 22, 1956, pp. 81-101

8. Highway Capacity Manual. TRB, National Research Council, Washington, D.C., 2000

9. Daganzo, C. F. Fundamentals of Transportation and Traffic Operations. Pergamon, Oxford, United Kingdom, 1997.

10. CORSIM User's Manual. FHWA, U.S. Department of Transportation, 2003.

11. Skabardonis, A., and N. Geroliminis. Real-Time Estimation of Travel Times Along Signalized Arterials. Presented at 16th International Symposium on Transportation and Traffic Theory, University of Maryland, July 2005.

The contents of this paper reflect the views of the authors, who are responsible for the facts and the accuracy of the data presented here. The contents do not necessarily reflect the official views or policy of the California Department of Transportation. This paper does not constitute a standard, specification, or regulation.

The Traffic Flow Theory and Characteristics Committee sponsored publication of this paper. 